\title{
Statistics and Probability of Detection in Wind Turbine Radar Clutter
}

\author{
S. Sinthuja*, S.V. Saravanan \\ Department of Electrical and Electronics Engineering (Marine), AMET University, Chennai, India \\ ${ }^{*}$ Corresponding author, e-mail: eei@journal.uad.ac.id
}

\begin{abstract}
A wind develops inside the perceptible pathway of a radar not simply makes undesirable radar returns as false targets furthermore truly impacts the radar's ability to distinguish centres of excitement for the area of the farm. In this work, accurate RCS models of wind turbines are delivered in perspective of honest to goodness wind turbine mess estimations, and acknowledgement probabilities of Swerling-1 centres and moreover undesirable wind turbines are registered. Results appear differently about those gained by expecting that wind turbine chaos is Rayleigh (or exponentially) circled.
\end{abstract}

Keywords: statistical, probability of detection, wind turbine, radar

\section{Introduction}

With the worldwide wind energy development that achieved 318 GW limits in late 2013, the impedance from twist turbines with ever bigger sharp edges (a record 100 meter long detailed in [1]) has rapidly turned into an immense issue for present-day radars that use Doppler marks to recognise focuses from a mess. Incalculable reviews have been distributed in extensive writings and also grouped reports. In any case, inquisitively, there were just a modest bunch of papers that examined measurable demonstrating of wind turbine radar mess [2-3].

In this article, a few likelihood dispersion work (PDF) models have been explored, and the most reasonable model is proposed to portray the wind turbine mess insights. Real radar cross segment (RCS) estimations of wind turbines arranged in Fenner, N.Y., are utilised for every single measurable model exhibited in this paper. Points of interest of these opinions can be found in [4-5]. The model is then used to compute identification probabilities of Swerling-1 focuses and also undesirable twist turbines for a non-specific aviation authority (ATC) radar. Results are contrasted with those acquired given a Rayleigh distributed wind turbine mess display, which can be appeared to be lacking to describe the wind turbine impedance.

\section{Statistical Models of Wind Turbine Clutter}

The MATLAB statistical toolbox [6], especially the FITTEST limit, is used to test and fit several PDFs to data. The limit enrols most outrageous likelihood gage (MLE) of parameters of specific PDFs given by the toolbox. Exactly when the I and Q signals (from radar complex data) are zero-mean Gaussian (i.e., customary) voltages, the ensuing banner sufficiency is spread by the Rayleigh PDF and the looking at compel is passed on by the exponential PDF [7]. The locator is believed to be immediate (i.e., voltage ID) and the PDF fitting performed the banner voltage or the square base of radar cross fragment $(\sqrt{R C S})$. The analysis and review of the wind energy explain in The Past, Present and Future of the Offshore Wind Power Technology-A Review [8]. The straight discoverer assumption is genuinely self-confident since an equal probability of area results got if a square-law locator is typical, given the exponential (as opposed to Rayleigh) PDF used for the target and uproar control bits of knowledge.

$$
T=Z \sqrt{\frac{v}{V}}
$$

where $Z$ is typically distributed with the expected value of 0 and variance $1 ; \mathrm{V}$ has a chi-squared distribution with $v$ degrees of freedom, and $Z$ and $V$ are independent. 


$$
f_{T}(t ; \nu)=\frac{\Gamma\left(\frac{\nu+1}{2}\right)}{\sqrt{\nu \pi} \Gamma\left(\frac{\nu}{2}\right)}\left(1+\frac{t^{2}}{\nu}\right)^{-\frac{\nu+1}{2}}
$$

where $v$ is the number of degrees of liberty and $\Gamma$ is the gamma function. For the case of $v=\infty$, Student t-distribution becomes the standard Normal distribution. The wind energy supported vehicles are explained in the Navigational \& safety assessment of wind farm support vessels [9].

\section{Spatial Extent of Wind Turbine Interference}

The spatial degree of wind turbine obstruction can evaluate as delineated in Figure 1. A wind turbine that is at the same range from the objective of intrigue can meddle with it as far out as $-27 \mathrm{~dB}$ side flap picks up in both azimuth headings. The wind turbine obstruction vitality will fall into the same determination cell from the objective utilising the radar's side projections and, in this manner, must be considered in the displaying. Those turbines that are not in a similar range container as the objective, however, fall into the reference window of the radar's Constant False Alarm Rate (CFAR) identifier or the locale limited by the red lines (as appeared in the figure) around the objective of intrigue may likewise meddle with the actual location. The spatial degree of this kind of impedance is the subject of another review.

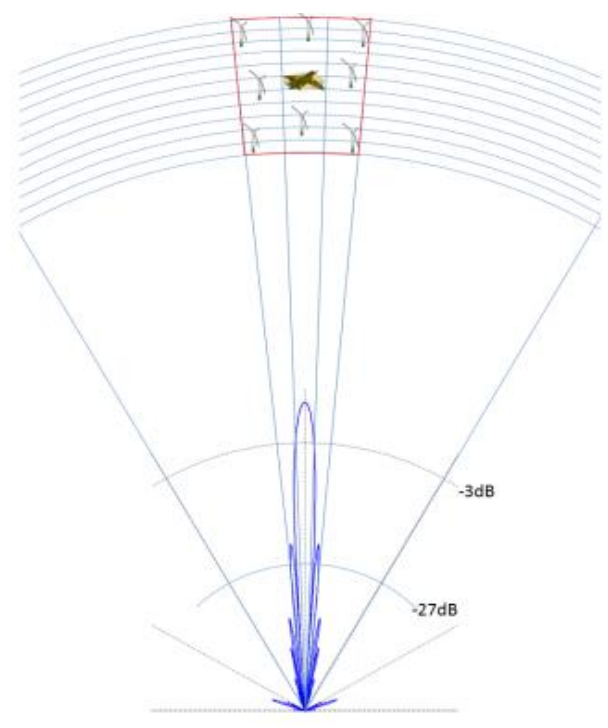

Figure 1. Depiction of impacted areas based on a probability of detection calculations

\section{Results and Discussions}

All probability of acknowledgement estimations is performed tolerating a Swerling-1 concentrate on, a likelihood of area (Pd) of $90 \%$, and a false ready rate (PFA) of 10-6 for one square meter centre up to the biggest extent of $80 \mathrm{NM}$. The goal is believed to be always perceived by methods for the apex of gathering mechanical assembly get and arranged at an indistinct range from an intruding wind turbine, which may be recognised in the essential projection or a side fold. The $0-\mathrm{dB}$ wind turbine gets addresses acknowledgement of a bend turbine at the zenith of the primary shaft, and lower get qualities indicate revelations at off bore find focuses that consolidate the tails of the standard fold or a side projection. The wind turbine hindrance is relied upon to occur at around $20 \mathrm{NM}$ from an L-band radar working at $750 \mathrm{~Hz}$ centre repeat. The wind turbine RCS dataset used for the estimation is that showed up in Figure 2. For this dataset, the turbine rotor is standing up to the radar at an azimuth purpose of 352。 $(0 \circ$ and 360 ० being particularly going up against the radar).

Figure 2 demonstrates the location of results comes about for both the objective $(\mathrm{Pd})$ and the meddling wind turbine (Pd turbine) as an element of the turbine picks up. The likelihood 
of discovery is figured given the wind turbine measurable model as portrayed by the t LocationScale.

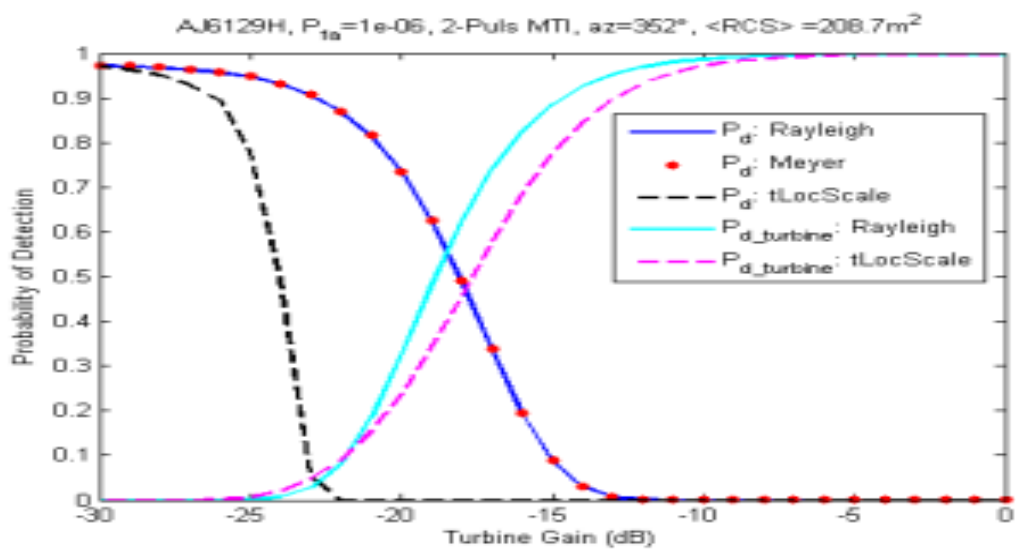

Figure 2. Probability of detection as a function of wind turbine gain

\section{Conclusions}

Statistical models of wind turbine RCS have been created in light of real estimations of turbine location probabilities of Swerling-1 focuses, and also undesirable wind turbines have been ascertained. It was demonstrated that the t Location-Scale PDF gave a solid match to the watched RCS insights of twist turbines under shifting degrees of clamour and target flag commitments. Other PDF models have likewise been analysed yet none were observed to be adequately satisfactory to demonstrate a blended flag with its parts showing diverse measurable practices or PDFs.

\section{References}

[1] Ancona D, McVeigh J. Wind Turbine-materials and Manufacturing Fact Sheet. Princeton Energy Resources International. 2001.

[2] Gallardo-Hernando B, Perez-Martinez F, Aguado-Encabo F. Statistical characterization of wind turbine clutter in C-band radars. In Radar International Conference on IEEE. September 2008: 360364.

[3] Muñoz-Ferreras JM, et al. Wind Turbine Clutter Observations and Theoretical Validation for Meteorological Radar Applications. IET radar, sonar \& navigation. 2011; 5(2): 111-117.

[4] Kent BM, et al. Dynamic radar cross section and radar Doppler measurements of commercial General Electric windmill power turbines Part 1: Predicted and measured radar signatures. IEEE Antennas and Propagation Magazine. 2008; 50(2): 211-219.

[5] Buterbaugh A. Dynamic Radar Cross Section and Radar Doppler Measurements of Commercial General Electric Windmill Power Turbines Part 2-Predicted and Measured Doppler Signatures. In Proceedings of the Symposium of the Antenna Measurement Technology Association. 2007.

[6] Siswayudi Azhari. Development of System Tracer of Lost Aircraft. International Journal of Informatics and Communication Technology (IJ-ICT). 2016; 5(2): 68-72.

[7] A. H. Sharief, M. Satya Sai Ram. An Improved ICI Self Cancellation Scheme for OFDM Systems Under Various Channels. International Journal of Electrical and Computer Engineering. April 2016; 6(2): 690-699.

[8] Patel AKP, Sivaraman A. The Past, Present and Future of the Offshore Wind Power Technology-A Review. In Offshore Technology Conference. Offshore Technology Conference. May 2016.

[9] Gopinath S. Navigational \& safety assessment of wind farm support vessels, RINA, Royal Institution of Naval Architects-Design and Operation of Wind Farm Support Vessels. Papers. 2015: 43-46.

[10] S.M. Mohiuddin, M.R.I. Sheikh. Stabilization of Solar-Wind Hybrid Power System by Using SMES. International Journal of Electrical and Computer Engineering. June 2014; 4(3): 351-358. 